\title{
Skeletal muscle assessment to understand cardiometabolic interactions
}

\author{
Vidhya Kumar ${ }^{1 *}$, Henry Chang ${ }^{1}$, Suzanne Smart ${ }^{1}$, Beth McCarthy' ${ }^{1}$ Ning Jin², Subha V Raman ${ }^{1}$ \\ From 19th Annual SCMR Scientific Sessions \\ Los Angeles, CA, USA. 27-30 January 2016
}

\section{Background}

Patients with diabetes and metabolic disorders have excess mortality after myocardial infarction (MI). Their mitochondrial function is often abnormal, and can be measured with phosphorus magnetic resonance spectroscopy (PMRS). Participation in a program of cardiac rehabilitation and secondary prevention (CRSP) reduces post-MI mortality, but typically involves only aerobic exercise and may not sufficiently improve mitochondrial function. An integrated assessment of skeletal muscle would potentially be useful to assess the impact of aerobic plus resistive exercise post-MI.

\section{Methods}

We tested a combined MR-based protocol with: 1) PMRS of quadriceps muscle at rest, during 30s of isometric quadriceps exercise, and during recovery and 2) quadriceps muscle fat quantification using a multi-echo Dixon sequence at 1.5 Tesla (Siemens, Erlangen). After shimming, an unlocalized FID sequence using the following parameters was used to acquire ${ }^{31} \mathrm{P}$ spectra: $\mathrm{TR}$ $=1000 \mathrm{~ms}, \mathrm{TE}=0.34 \mathrm{~ms}, \mathrm{BW}=2000 \mathrm{~Hz}$, points $=$ 1024 , averages $=4$. Fat $/$ water quantification was acquired with: TR $=11.1 \mathrm{~ms}, 6$ echoes with TE minimized, $\mathrm{BW}=1150 \mathrm{~Hz}$, slice thickness $=4 \mathrm{~mm}$.

$\mathrm{PCr}$ peak amplitudes, representing concentration, were quantified using jMRUI (Lyons, France) and recovery time was calculated with a best-fit mono-exponential function (Fig 1A). Quantitative fat maps were generated from the Dixon sequence, with pixel intensity representing fat percentage (Fig 1C). Feasibility was assessed in healthy volunteers and patients starting a CRSP program post-MI.

\section{Results}

Nine volunteers and 15 patients were enrolled. Left ventricular ejection fraction was preserved in CRSP patients $(56 \pm 10 \%)$. Maximum exertion ability, measured before starting CRSP was similar in diabetic and non-diabetic patients $(3.05 \pm 0.6$ vs. $3.4 \pm 0.8$ metabolic equivalents [METs], $\mathrm{p}=0.4$ ). Hba1c averaged $7.8 \%$ in diabetics whose LDL levels averaged $109.7 \pm 51.5$ vs. $106.7 \pm 33.4$ $\mathrm{mg} / \mathrm{dL}$ in nondiabetics $(\mathrm{p}=0.9)$. $\mathrm{PCr}$ recovery time was longer $(41.9 \pm 1.4$ vs. $32.1 \pm 7.4 \mathrm{~s}, \mathrm{p}=0.05)$, and intramuscular fat percentage higher in CRSP patients vs. controls $(8.7 \pm 2.9$ vs. $2.54 \pm 0.6 \%, \mathrm{p}<0.001)$ (Fig $1 \mathrm{~B}$, D). Intramuscular fat percentage was similar in diabetic and non-diabetic patients prior to starting CRSP ( $\mathrm{p}=0.4$ ), and $\mathrm{PCr}$ recovery time tended to be longer in diabetic patients compared to nondiabetic patients and controls ( $\mathrm{p}=0.03$ for trends across groups). Preliminary follow-up data suggest considerably worse improvement in METs in diabetic vs. nondiabetic patients (delta $=1.0$ \pm 0.8 vs. $4.0 \pm 2.4, \mathrm{p}=0.06$ ).

\section{Conclusions}

An integrated protocol of skeletal muscle 31P spectroscopy with fat quantification is feasible in patients starting cardiac rehabilitation, and may help improve understanding of cardiometabolic interactions that are not evident from cardiac measures alone.

\section{Authors' details \\ ${ }^{1}$ Ohio State University, Columbus, OH, USA. ${ }^{2}$ Siemens Medical Solutions, Columbus, OH, USA.}

Published: 27 January 2016

Ohio State University, Columbus, OH, USA

Full list of author information is available at the end of the article 

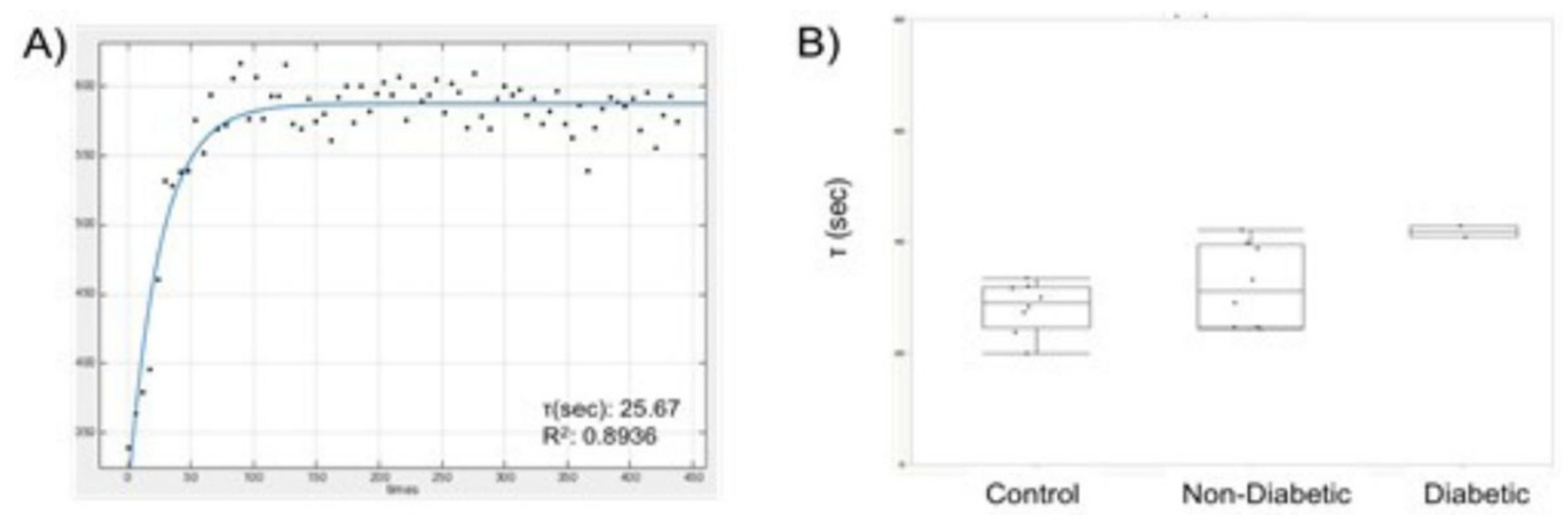

\section{C)}

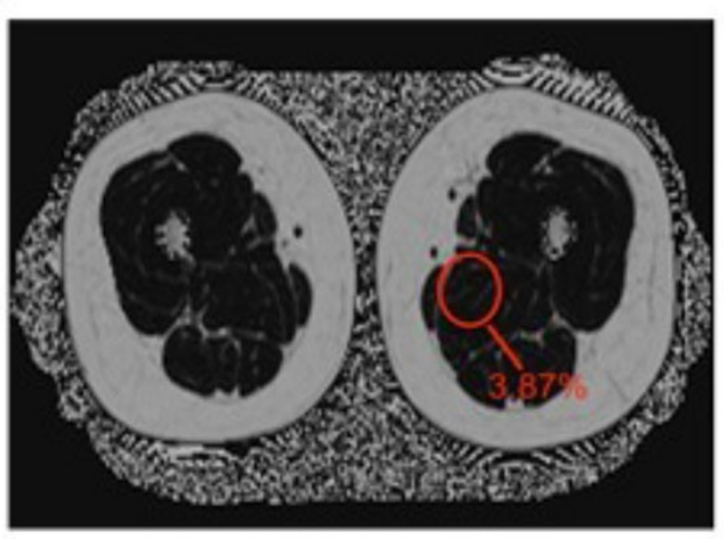

D)

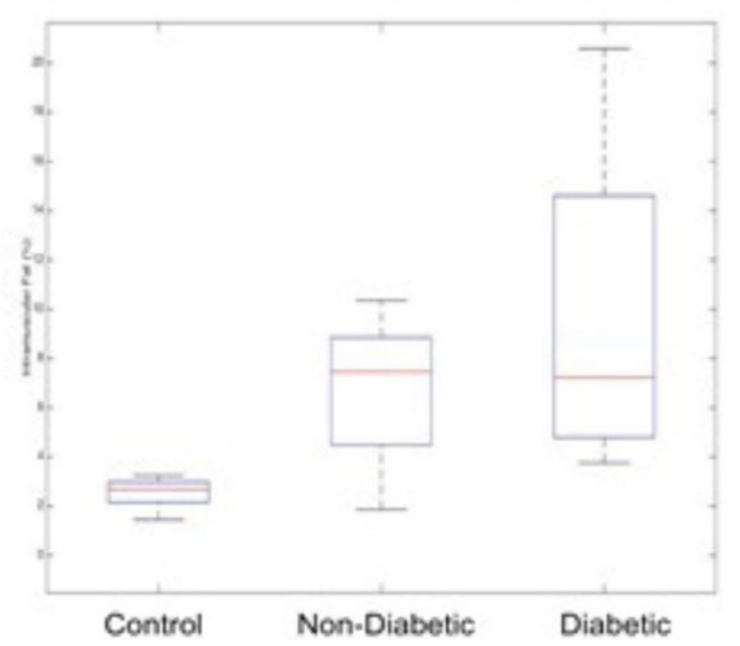

Figure $1 \mathrm{~A}$ ) Phosphocreatine ( $\mathrm{PCr}$ ) concentration is depleted with rapid resistive extremity exercise and returns to baseline levels within a recovery period $(\tau)$, which is a well-established biomarker of mitochondrial oxidative function. A representative recovery curve is shown. B) Comparison of $\mathrm{PCr}$ recovery times demonstrates sequentially poorer mitochondrial oxidative capacity in control subjects, nondiabetic and diabetic patients. C) A representative quantitative fat image acquired through the leg illustrates the measurement of intramuscular lipid. D) Comparison of results indicates sequentially higher skeletal muscle fat content in control subjects, non-diabetic and diabetic patients.

doi:10.1186/1532-429X-18-S1-P220

Cite this article as: Kumar et al:: Skeletal muscle assessment to understand cardiometabolic interactions. Journal of Cardiovascular Magnetic Resonance 2016 18(Suppl 1):P220.

\section{Submit your next manuscript to BioMed Central} and take full advantage of:

- Convenient online submission

- Thorough peer review

- No space constraints or color figure charges

- Immediate publication on acceptance

- Inclusion in PubMed, CAS, Scopus and Google Scholar

- Research which is freely available for redistribution

Submit your manuscript at www.biomedcentral.com/submit
C Biomed Central 